\title{
Monoclonal gammopathy with renal significance
}

\author{
Corresponding author: \\ Jarosław Czyż \\ Department of Haematology \\ University Hospital No. 2 \\ - Jan Biziel Memorial Hospita \\ Ujejskiego 75 St. \\ 85-168 Bydgoszcz, Poland \\ E-mail: jczyz@onet.p
}

\begin{abstract}
Monoclonal gammopathy of unknown significance (MGUS) is a benign condition that carries a risk of progression to haematological malignancy. It is accepted that MGUS should not be treated until progression to multiple myeloma or another lymphoid malignancy. Recently, growing evidence has started to show that even small monoclonal clones can be responsible for renal impairment. Long-term observation of patients with monoclonal gammopathy and abnormal renal function showed that this condition can significantly affect renal and overall survival. Patients with monoclonal gammopathy with renal impairment have also higher risk of relapse after kidney transplantation. Among patients with monoclonal gammopathy of unknown significance there is a group of defined monoclonal component-related diseases, which includes: light-chain amyloidosis, monoclonal immunoglobulin deposition disease, crystal-storing histiocytosis, cryoglobulinaemias, and some others. They can be diagnosed on the base of clinical features and on histological examination. In patients with monoclonal protein and deposition of fragments or whole particle of monoclonal immunoglobulin with distinct localisation and substructural organisation can be found. The treatment strategy is targeting of $\mathrm{B}$ cell clones, which requires administration of chemotherapeutics or other medications that are used for the treatment of lymphoid malignancy or myeloma. The choice of therapeutic agent should take into account the current kidney status. Treatment of renal disease should not differ from other patients with similar conditions not related to monoclonal protein. The expert opinion is that the presence of monoclonal gammapathy is not a contraindication to kidney transplantation. Key words: monoclonal gammopathy, renal failure, treatment
\end{abstract}

Med Res J 2016; 1 (2): 53-57

\section{Introduction}

Monoclonal gammopathy of unknown significance (MGUS) is defined by a monoclonal immunoglobulin in serum and $10 \%$ or fewer plasma cells in the bone marrow, in persons without evidence of multiple myeloma, amyloidosis, or other lymphoproliferative B-cell disorders [1, 2]. It is a benign condition that carries a risk of progression to a haematological malignancy. The probability of developing multiple myeloma, Waldenstrom's macroglobulinaemia, amyloidosis, or any other lymphoproliferative disorder in patients with MGUS is approximately $1 \%$ per year [2]. It is generally accepted that the condition should not be treated until progression to multiple myeloma with organ damage, or other lymphoid malignancies. Nephrotic complications in patients with multiple myeloma usually result from cast nephropathy, which is caused by excessive production of light chains. High tumour mass does not necessarily increase the risk of kidney damage [3]. The evidence from animal experiments shows that injection of even a small amount of Bence-Jones protein isolated from serum of myeloma patients can induce cast nephropathy [4]. There is a growing amount of evidence that proves that even small monoclonal clones can be responsible for renal impairment in affected patients [5]. Despite their non-malignant nature they can cause significant morbidity or even premature death. The affected patients are still diagnosed as having MGUS, but the presence of monoclonal protein is no longer unknown. To avoid confusion, the term glomerulonephritis with MGUS or monoclonal immunoglobulin deposition disease (MIDD) with MGUS started to be replaced by the term monoclonal gammopathy of renal significance (MGRS) [6]. Despite evidence that links the presence of monoclonal immunoglobulin in serum to kidney impairment, many patients with monoclonal gammopathy, not fulfilling the criteria of myeloma, remain untreated or 
undertreated. In a published report from Italy, among patients diagnosed with light chain deposition disease with renal involvement, more than a quarter did not receive any treatment [7]. In another report from Mayo, the long-term observation of patients with the same diagnosis showed that the overall five-year survival rate was only $70 \%$. The five-year renal survival was 37\% [8]. What is worse, patients with MGRS are at higher risk of relapse after kidney transplantation $[9,10]$.

Apart from monoclonal gammopathy of unknown significance there is a group of defined monoclonal component diseases which if left untreated may cause irreversible kidney damage or may even be fatal. They include light-chain $(\mathrm{AL})$ amyloidosis, monoclonal immunoglobulin deposition disease, crystal-storing histiocytosis, and cryoglobulianemias.

\section{Diagnosis}

\section{Microscopic examination}

In most cases MGRS results from deposition of fragments or whole particles of monoclonal immunoglobulin in distinct localisations and substructural organisation. They can aggregate causing glomerulopathies with organised amyloid fibrils or microtubular deposits as well as form punctate aggregates, produce crystals, or cause vascular obstruction [5]. Amyloid fibrils are most commonly seen in $\mathrm{AL}$ amyloidosis, immunoglobulin heavy chain, or immunoglobulin light and heavy chain amyloidosis [11]. Microtubular monoclonal immunoglobulin deposits, which are responsible for vascular obstruction in small vessels, are usually seen in type I and type II cryoglobulinaemias or immunocytoid glomerulopathy. Non-organised deposits (Randal and non-Randal type) are observed in patients with monoclonal immunoglobulin deposition disease or in proliferative glomerulonephritis with deposits of monoclonal immunoglobulins [12, 13]. Monoclonal gammopathy of renal significance can be responsible for the tubular defect present in Fanconi syndrome [14]. In this case the defect is caused by crystal deposits.

\section{Clinical features}

The results of the study performed in Mayo showed that more than half of the patients with monoclonal gammopathy had renal lesions that were directly related to the presence of monoclonal protein [15]. Other causes included diabetic nephropathy, focal segmental glomerulosclerosis, and arterionephrosclerosis. Therefore, the diagnosis of MGRS should be based on the detection of monoclonal protein in the kidney. Renal biopsy should be performed for every patient with the presence of monoclonal protein, renal insufficiency, and severe proteinuria. Immunofluorescence and electron microscopy are essential to establish the type of heavy and light chain monoclonal immunoglobulin deposits in the kidney, as well as the pattern of their organisation [16]. Immunofixation and protein electrophoresis should be performed. If serum or urine immunofixation is negative for monoclonal protein, measurement of serum free light chain ratio can help with diagnosis. Bone marrow examination is also an essential part of the diagnostic process. Plasmocyte or lymphocyte clonality should be established. The type of monoclonal protein should be the same as that found in renal deposits and in serum [6].

In many cases of MGRS the clinical diagnosis is of AL amyloidosis - a progressive condition characterised by tissue and organ amyloid deposits. It is usually associated with small numbers of clonal plasma cells that produce $\lambda$ light chains present in the bone marrow [17]. The general symptoms include weight loss, fatigue, or oedema. On physical examination, characteristic abnormalities include enlargement of the tongue, periorbital purpura, or periarticular amyloid infiltration. Patients with amyloidosis may also present with hepatomegaly or carpal tunnel syndrome [18].The most commonly affected organs are: kidney, heart, and liver as well as the peripheral and autonomic nervous systems. At the time of diagnosis nephrotic syndrome is present in $50 \%$ of patients, and kidney failure in $18 \%$ of patients [5]. The diagnosis of AL should always be confirmed by biopsy. The recommended procedure for this is a fine-needle aspiration of abdominal fat or minor labial salivary gland. The liver or kidney should be considered as organs of second choice for biopsy because of minor risk of bleeding $[18,19]$. Amyloid deposits stain positive with Congo red and demonstrate apple-green birefringence under polarised light [20].

Monoclonal immunoglobulin deposition disease includes: light-chain deposition disease, light- and heavy-chain deposition disease, and heavy-chain deposition disease. Kappa light-chains are more frequent in MIDD than in AL amyloidosis. Typical manifestation of renal involvement includes massive proteinuria, haematuria, hypertension, and premature kidney failure [16, 21]. Light-chain deposition disease (LCDD) and $\mathrm{AL}$ amyloidosis have several features in common: both may affect the heart, liver, or peripheral nervous system and may cause kidney failure. Most patients with LCDD already present symptoms of renal failure at the time of initial presentation of the disease. Kidney biopsy shows deposits that are Congo red negative, amorphous, and electron dense [22]. In some situations both amyloid and nonfibrillar monoclonal light-chain deposits may be present in the same patient $[20,23]$.

Cryoglobulins are immunoglobulins that precipitate or gel reversibly at temperatures below $37^{\circ} \mathrm{C}$ [5]. Type I monoclonal cryoglobulinaemia is uncommon and is 
characterised by the presence of IgG immunoglobulin. The symptoms are typically caused by precipitation of the cryoglobulin in small vessels. Clinical presentation usually involves the skin (Raynaud phenomenon, acrocyanosis, necrosis, purpura and ulcers, cold urticaria) or manifests as nephropathy [24]. In contrast to patients with type II or type III cryoglobulinaemia, renal involvement has been described only sporadically [25]. Type II cryoglobulinaemia is usually associated with hepatitis $\mathrm{C}$ virus $(\mathrm{HCV})$ infection. It presents with monoclonal $\operatorname{lgM}$ and coexisting polyclonal IgG immunoglobulin. The monoclonal IgM rheumatoid factors bind the $F_{C}$ portion of IgG [26]. The clinical features are similar to those present in type I cryoglobulinaemia and also include purpura, neuropathy, and renal involvement. Additionally, weakness, arthralgia, and liver involvement can be observed in some patients [27].

\section{Proliferative glomerulonephritis with monoclonal immunoglobulin deposits}

Proliferative glomerulonephritis with monoclonal immunoglobulin deposits presents with a non-organised deposition of whole $\operatorname{lgG} 3 \kappa$ immunoglobulin particles. Typical symptoms include proteinuria and progressive chronic kidney disease [28]. Other organs, including bone marrow, are rarely affected.

\section{Adult Fanconi syndrome}

Adult Fanconi syndrome is characterised by the presence of glycosuria, aminoaciduria, hypophosphataemia, and vitamin D-resistant rickets. It is caused by lysosomal accumulation of monoclonal kappa light chains, which aggregate and form crystals. It can be observed in patients with plasma cell or lymphoid proliferation [5, 29]. These patients may suffer from recurrent bone pain. This, in combination with the presence of light-chains in urine it may in some situations result in the diagnosis of BenceJones myeloma. It is therefore important to establish glucose levels in urine. Patients with Adult Falconi syndrome will have elevated glucose levels while their blood glucose levels remains normal [29]. Untreated disease may lead to kidney failure [14]. In some situations Fanconi syndrome can be a part of crystal-storing histiocytosis, with light-chain crystal accumulation in histiocytes in the bone marrow, spleen, and lymph nodes [30].

\section{Treatment}

\section{Elimination of monoclonal B clone}

There is no standard treatment of MGRS. Targeting a $B$ cell clone requires an administration of chemotherapeutics or other medications that are used for treatment of lymphoid malignancies or myelomas. The choice of the therapeutic agent should be made according to kidney function. Alkylating agents, such as melphalan must be used with caution. When possible, melphalan should be replaced by cyclophosphamide, which is better tolerated by patients with impaired renal function [31]. In terms of immunomodulators, thalidomide should be used as a first-line drug before lenalidomide because it does not require dose reduction in patients with impaired kidney function [32]. Fludarabine, an adenine nucleoside analogue, should be avoided in patients with severe renal impairment [32]. Bendamustine is not metabolised in the kidneys and may be given to patients with MGRS without modification due to renal function parameters [33, 34]. The use of monoclonal antibodies, including anty-CD20, is safe even for patients with impaired renal function $[35,36]$.

In younger patients with myeloma, the standard procedure consists of consolidation with high-dose melphalan chemotherapy, followed by autologous stem cell transplantation. This treatment can be administrated to patients with severe kidney dysfunction caused by MGRS, but significant morbidity and mortality must be taken into account $[37,38]$.

\section{Treatment of amyloidosis}

Treatment of amyloidosis should refer to the Mayo Clinic criteria [39]. The first-line treatment for patients in stage I and stage II should include bortezomib, steroids, and alkylating agents [40, 41]. The second-line treatment is based on thalidomide [42]. Certain patients can undergo a consolidation with high-dose melphalan chemotherapy followed by autologous stem cell transplantation [43, 44]. Stage III patients present a therapeutic challenge because of an extremely poor prognosis. Administration of antibodies to serum amyloid $\mathrm{P}$ component may be a successful treatment option in such a difficult clinical scenario [45].

\section{Monoclonal immunoglobulin deposition disease}

There is no standard treatment for this condition. The procedures are based on consensus recommendations. The majority of patients are treated with thalidomide or bortezomib. In some cases high-dose melphalan chemotherapy followed by HSCT may be implemented [21, 46-48]. There is a strong association between haematological response to chemotherapy and renal function [21]. Most of the patients who achieve a complete or partial response never require dialysis. In contrast, those with minimal or partial haematological response usually develop end-stage renal disease. According to the consensus recommendation, the main goal of the treatment for patients with early stage chronic kidney disease (CKD) 1-3 should be preservation of 
kidney function. Elimination of monoclonal B-clone should be the main target for patients with CKD 4-5, who are eligible for kidney transplantation. If kidney transplantation is not possible, the therapy should be aimed at preservation of extra-renal organs [16].

\section{Treatment of cryoglobulinaemia}

According to the recommendations for cryoglobulinaemia, asymptomatic patients require close monitoring of renal parameters [16]. Symptomatic patients with plasmocytic proliferation of monoclonal IgG or IgA protein should be treated as myeloma patients. Patients with lymphoplasmocytic proliferation and production of IgM monoclonal protein should receive Waldenstrom macroglobulinaemia type of treatment, including Rituximab, and chronic lymphocytic leukaemia-type clone should be treated as is recommended for patients with this condition. For patients with severely impaired kidney function, bendamustine (which is not eliminated by kidneys) could be effective [25, 49, 50]. Type II cryoglobulinaemia is usually caused by IgM monoclonal protein and in most cases is linked to hepatitis $C$ infection. It is recommended to introduce antiviral treatment for every patient with symptomatic type II cryoglobulinaemia and active hepatitis [16]. For patients without active viral replication, a wait and watch approach is recommended with Rituximab treatment when flares of the disease are observed [51].

\section{Proliferative glomerulonephritis with monoclonal immunoglobulin deposits}

Patients at an early stage of the disease (stage I-II, proteinuria less than $1 \mathrm{~g}$ per day) only require observation. In more advanced stages of the disease, cyclophosphamide or bortezomib treatment can be introduced. Consolidation with high-dose melphalan chemotherapy with autologous stem cell transplant is also possible. Complete haematological remission is the main target of the treatment for those awaiting kidney transplantation $[10,16]$.

\section{Adult Fanconi syndrome}

Myeloma-like chemotherapy (bortezomib-, thalidomide-, or cyclophosphamide-based) should be introduced in the early stages of the disease. The treatment of more advanced stages of the disease includes high-dose melphalan chemotherapy with autologous stem cell transplantation (before or after renal transplantation). Only symptomatic treatment should be offered to patients who are not eligible for kidney transplantation [16].

\section{Response assessment}

It is logical and recommended to adoptdisease response assessment in conditions related to lightchain proliferation criteria introduced for amyloidosis assessment [16]. This system includes four levels of responses: complete response: normal free light chain ratio and negative serum and urine immunofixation, very good partial response (with difference between involved and uninvolved free-light chain $<40 \mathrm{mg} / \mathrm{L}$ ), partial response (decrease in free-light chain $>50 \%$ ), and no response [52]. In diseases caused by whole monoclonal immunoglobulin particle, assessment should be based on International uniform response criteria for multiple myeloma [16, 53]. Another recommendation is to reintroduce treatment immediately after the reappearance of monoclonal immunoglobulin, before kidney parameters decrease [16].

\section{Treatment of renal failure}

The treatment of renal failure should not differ from the treatment of other kidney conditions that are not related to the presence of monoclonal protein. According to expert opinion, monoclonal gammopathy is not a contraindication to kidney transplantation. Appropriate treatment should be introduced to achieve the best monoclonal protein response before the transplantation. The response is directly correlated to kidney transplant survival and should be reintroduced immediately after reappearance of monoclonal protein.

\section{References}

1. The International Myeloma Working G. Criteria for the classification of monoclonal gammopathies, multiple myeloma and related disorders: a report of the International Myeloma Working Group. Br J Haematol 2003; 121: 749-757.

2. Kyle RA, Durie BG, Rajkumar SV et al. Monoclonal gammopathy of undetermined significance (MGUS) and smoldering (asymptomatic) multiple myeloma: IMWG consensus perspectives risk factors for progression and guidelines for monitoring and management. Leukemia 2010; 24: 1121-1127.

3. Bridoux F, Fermand JP. Optimizing treatment strategies in myeloma cast nephropathy: rationale for a randomized prospective trial. Adv Chronic Kidney Dis 2012; 19: 333-341.

4. Solomon A, Weiss DT, Kattine AA. Nephrotoxic potential of Bence Jones proteins. N Engl J Med 1991; 324: 1845-1851.

5. Merlini G, Stone MJ. Dangerous small B-cell clones. Blood 2006; 108: 2520-2530.

6. Leung N, Bridoux F, Hutchison CA et al. Monoclonal gammopathy of renal significance: when MGUS is no longer undetermined or insignificant. Blood 2012; 120: 4292-4295.

7. Pozzi C, D'Amico M, Fogazzi GB et al. Light chain deposition disease with renal involvement: clinical characteristics and prognostic factors. Am J Kidney Dis 2003; 42: 1154-1163.

8. Heilman RL, Velosa JA, Holley KE et al. Long-term follow-up and response to chemotherapy in patients with light-chain deposition disease. Am J Kidney Dis 1992; 20: 34-41.

9. Leung N, Lager DJ, Gertz MA et al. Long-term outcome of renal transplantation in light-chain deposition disease. Am J Kidney Dis 2004; 43: 147-153. 
10. Lorenz EC, Sethi S, Leung $\mathrm{N}$ et al. Recurrent membranoproliferative glomerulonephritis after kidney transplantation. Kidney Int 2010; 77 721-728

11. Touchard G. Ultrastructural pattern and classification of renal monocIonal immunoglobulin deposits. In Monoclonal gammopathies and the kidney. Springer 2003; 95-117.

12. Ronco P, Aucouturier P, Mougenot B. Monoclonal gammopathies: multiple myeloma, amyloidosis, and related disorders. Diseases of the Kidney 2001: 409-410

13. Vrana JA, Gamez JD, Madden BJ et al. Classification of amyloidosis by laser microdissection and mass spectrometry-based proteomic analysis in clinical biopsy specimens. Blood 2009; 114: 4957-4959.

14. Lacy $M Q$, Gertz MA. Acquired Fanconi's syndrome associated with monoclonal gammopathies. Hematol Oncol Clin North Am 1999; 13: 1273-1280.

15. Paueksakon P, Revelo MP, Horn RG et al. Monoclonal gammopathy: significance and possible causality in renal disease. Am J Kidney Dis 42: 87-95.

16. Fermand JP, Bridoux F, Kyle RA et al. How I treat monoclonal gammopathy of renal significance (MGRS). Blood 2013; 122: 3583-3590.

17. Comenzo RL, Wally J, Kica G et al. Clonal immunoglobulin light chain variable region germline gene use in $\mathrm{AL}$ amyloidosis: association with dominant amyloid-related organ involvement and survival after stem cell transplantation. Br J Haematol 1999; 106: 744-751.

18. Merlini G, Wechalekar AD, Palladini G. Systemic light chain amyloidosis: an update for treating physicians. Blood 2013; 121: 5124-5130.

19. Obici L, Perfetti V, Palladini G et al. Clinical aspects of systemic amyloid diseases. Biochim Biophys Acta 2005; 1753: 11-22.

20. Gertz MA, Comenzo R, Falk RH et al. Definition of organ involvement and treatment response in immunoglobulin light chain amyloidosis $(A L)$ : A consensus opinion from the 10th International Symposium on Amyloid and Amyloidosis. Am J Hematol 2005; 79: 319-328

21. Sayed RH, Wechalekar AD, Gilbertson JA et al. Natural history and outcome of light chain deposition disease. Blood 2015; 126: 2805-2810.

22. Khamlichi A, Rocca A, Touchard $G$ et al. Role of light chain variable region in myeloma with light chain deposition disease: evidence from an experimental model. Blood 1995; 86: 3655-3659.

23. Kaplan B, Vidal R, Kumar A et al. Amino-terminal identity of co-existent amyloid and non-amyloid immunoglobulin $\kappa$ light chain deposits. A human disease to study alterations of protein conformation. Clinical \& Experimental Immunology 1997; 110: 472-478.

24. Néel A, Perrin F, Decaux O et al. Long-term outcome of monoclona (type 1) cryoglobulinemia. Am J Hematol 2014; 89: 156-161.

25. Karras A, Noël L-H, Droz D et al. Renal involvement in monoclonal (type I) cryoglobulinemia: Two cases associated with IgG3 kappa cryoglobulin. Am J Kidney Dis 2002; 40: 1091-1096.

26. Stone MJ, McElroy YG, Pestronk A et al. Human monoclonal macroglobulins with antibody activity. Semin Oncol 2003; 30: 318-324.

27. Ferri C, Sebastiani M, Giuggioli D et al. Mixed cryoglobulinemia: demographic, clinical, and serologic features and survival in 231 patients. Seminars in Arthritis and Rheumatism 2004; 33: 355-374

28. Nasr SH, Satoskar A, Markowitz GS et al. Proliferative Glomerulonephritis with Monoclonal IgG Deposits. J Am Soc Nephrol 2009; 20 : 2055-2064

29. Gertz M, Buadi FK. Case vignettes and other brain teasers of monocIonal gammopathies. Hematology Am Soc Hematol Educ Program 2012; 2012: 582-585

30. El Hamel C, Thierry A, Trouillas P et al. Crystal-storing histiocytosis with renal Fanconi syndrome: pathological and molecular characteristics compared with classical myeloma-associated Fanconi syndrome. Nephrol Dial Transplant 2010; 25: 2982-2990

31. Carlson K, Hjorth M, Knudsen LM, The Nordic Myeloma Study G. Toxicity in standard melphalan-prednisone therapy among myeloma patients with renal failure - a retrospective analysis and recommendations for dose adjustment. Br J Haematol 2005; 128: 631-635.

32. Lichtman SM, Etcubanas E, Budman DR et al. The pharmacokinetics and pharmacodynamics of fludarabine phosphate in patients with renal impairment: a prospective dose adjustment study. Cancer Invest 2002; 20: 904-913.

33. Ramasamy K, Hazel B, Mahmood S et al. Bendamustine in combination with thalidomide and dexamethasone is an effective therapy for myeloma patients with end stage renal disease. $\mathrm{Br} \mathrm{J}$ Haematol 2011; 155: 632-634.
34. Pönisch W, Andrea M, Wagner I et al. Successful treatment of patients with newly diagnosed/untreated multiple myeloma and advanced renal failure using bortezomib in combination with bendamustine and prednisone. J Cancer Res Clin Oncol 2012; 138: 1405-1412.

35. Jillella AP, Dainer PM, Kallab AM, Ustun C. Treatment of a patient with end-stage renal disease with Rituximab: Pharmacokinetic evaluation suggests Rituximab is not eliminated by hemodialysis. Am J Hematol 2002; 71: 219-222.

36. Vieira CA, Agarwal A, Book BK et al. Rituximab for reduction of anti-HLA antibodies in patients awaiting renal transplantation: 1. Safety, pharmacodynamics, and pharmacokinetics1. Transplantation 2004; 77: 542-548

37. Schrier RW, Parikh CR. Comparison of renal injury in myeloablative autologous, myeloablative allogeneic and non-myeloablative allogeneic haematopoietic cell transplantation. Nephrol Dial Transplant 2005; 20: 678-683

38. Irazabal MV, Eirin A, Gertz MA et al. Acute kidney injury during leukocyte engraftment after autologous stem cell transplantation in patients with light-chain amyloidosis. Am J Hematol 2012; 87: 51-54.

39. Kumar S, Dispenzieri A, Lacy MQ et al. Revised Prognostic Staging System for Light Chain Amyloidosis Incorporating Cardiac Biomarkers and Serum Free Light Chain Measurements. J Clin Oncol 2012; 30: 989-995.

40. Venner CP, Lane T, Foard D et al. Cyclophosphamide, bortezomib, and dexamethasone therapy in $\mathrm{AL}$ amyloidosis is associated with high clonal response rates and prolonged progression-free survival. Blood 2012; 119: 4387-4390.

41. Mikhael JR, Schuster SR, Jimenez-Zepeda VH et al. Cyclophosphamide-bortezomib-dexamethasone (CyBorD) produces rapid and complete hematologic response in patients with AL amyloidosis. Blood 2012: 119: 4391-4394.

42. Venner CP, Gillmore JD, Sachchithanantham S et al. A matched comparison of cyclophosphamide, bortezomib and dexamethasone (CVD) versus risk-adapted cyclophosphamide, thalidomide and dexamethasone (CTD) in AL amyloidosis. Leukemia 2014; 28: 2304-2310.

43. Cibeira MT, Sanchorawala V, Seldin DC et al. Outcome of AL amyloidosis after high-dose melphalan and autologous stem cell transplantation: long-term results in a series of 421 patients. Blood 2011; 118: 4346-4352.

44. D'Souza A, Dispenzieri A Wirk B et al. Improved Outcomes After Autologous Hematopoietic Cell Transplantation for Light Chain Amyloidosis: A Center for International Blood and Marrow Transplant Research Study. Journal of Clinical Oncology 2015; 33: 3741-3749 .

45. Richards DB, Cookson LM, Berges AC et al. Therapeutic Clearance of Amyloid by Antibodies to Serum Amyloid P Component. N Engl J Med 2015: 373: 1106-1114.

46. Weichman K, Dember LM, Prokaeva T et al. Clinical and molecular characteristics of patients with non-amyloid light chain deposition disorders, and outcome following treatment with high-dose melphalan and autologous stem cell transplantation. Bone Marrow Transplant 2006; 38: 339-343.

47. Lorenz EC, Gertz MA, Fervenza FC et al. Long-term outcome of autologous stem cell transplantation in light chain deposition disease. Nephrol Dial Transplant 2008; 23: 2052-2057.

48. Kastritis E, Migkou M, Gavriatopoulou M et al. Treatment of light chain deposition disease with bortezomib and dexamethasone. Haematologica 2009; 94: 300-302.

49. Ramos-Casals M, Stone JH, Cid MC, Bosch X. The cryoglobulinaemias. Lancet 2012; 379: 348-360.

50. Terrier B, Krastinova E, Marie I et al. Management of noninfectious mixed cryoglobulinemia vasculitis: data from 242 cases included in the CryoVas survey. Blood 2012; 119: 5996-6004.

51. Dammacco F, Tucci FA, Lauletta G et al. Pegylated interferonribavirin, and rituximab combined therapy of hepatitis $C$ virus-related mixed cryoglobulinemia: a long-term study. Blood 2010; 116: 343-353.

52. Palladini G, Dispenzieri A, Gertz MA et al. New Criteria for Response to Treatment in Immunoglobulin Light Chain Amyloidosis Based on Free Light Chain Measurement and Cardiac Biomarkers: Impact on Survival Outcomes. J Clin Oncol 2012; 30: 4541-4549.

53. Durie BGM, Harousseau JL, Miguel JS et al. International uniform response criteria for multiple myeloma. Leukemia 2006; 20: 1467-1473. 